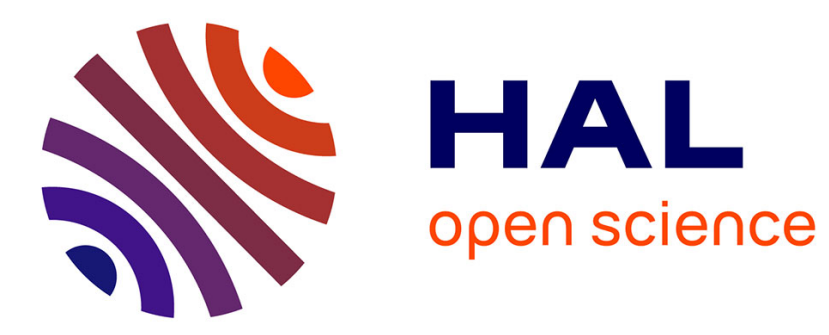

\title{
Ne rien attendre de la fortune ou la disqualification cartésienne de l'aventure
}

\author{
François-Xavier de Peretti
}

\section{To cite this version:}

François-Xavier de Peretti. Ne rien attendre de la fortune ou la disqualification cartésienne de l'aventure. Litttératures classiques, 2019. hal-02294673

\section{HAL Id: hal-02294673 https://hal-amu.archives-ouvertes.fr/hal-02294673}

Submitted on 23 Sep 2019

HAL is a multi-disciplinary open access archive for the deposit and dissemination of scientific research documents, whether they are published or not. The documents may come from teaching and research institutions in France or abroad, or from public or private research centers.
L'archive ouverte pluridisciplinaire HAL, est destinée au dépôt et à la diffusion de documents scientifiques de niveau recherche, publiés ou non, émanant des établissements d'enseignement et de recherche français ou étrangers, des laboratoires publics ou privés. 
François-Xavier de Peretti

\section{Ne rien attendre de la fortune ou la disqualification cartésienne de l'aventure}

Parmi la vaste galerie des portraits de Descartes, celui d'un Descartes aventurier voire belliqueux tient une bonne place, soit qu'on se plaise à saluer sa décision de sortir de la sujétion de ses précepteurs et de quitter l'étude des lettres pour se mettre à voyager et pour se plonger dans "le grand livre du monde ", soit qu'on veuille rendre hommage à l'héroïsme de sa pensée. Il est vrai qu’il aura entrepris de voyager en s'engageant en 1618 comme soldat dans l'armée protestante de Maurice de Nassau en Hollande, puis en 1619 sous la bannière de l'armée catholique du duc Maximilien de Bavière en Allemagne. Il n'en est pas moins vrai qu'il combattra intellectuellement sur le double front du scepticisme renaissant et de la scolastique déclinante, comparant ceux qui découvrent peu à peu la vérité dans les sciences à ces chefs d'armée prenant villes et provinces et dont les forces ont coutume de crôitre à proportion des victoires²

Jean-Louis Guez de Balzac, dans une lettre du 30 mars 1628 rappelant à Descartes sa promesse de publier l'histoire de son esprit, n'hésite pas à utiliser le terme d'aventure et à présenter la réforme cartésienne de la physique et de la philosophie comme une lutte héroïque, voire homérique, contre les scolastiques, l'assurant qu'il y aura plaisir à lire ses « diverses aventures dans la moyenne et dans la plus haute région de l'air » et à considérer ses "prouesses contre les Géants de l'École ${ }^{3}$ ». Et de «ce cavalier français qui partit d'un si bon pas ${ }^{4}$ ", Hegel encore saluera l'héroïsme et l'extraordinaire hardiesse d'avoir pris le risque de tout recommencer depuis les fondements dans l'ordre de la connaissance. ${ }^{5}$. Il n'est cependant pas superflu de se demander jusqu'où cette légende cartésienne s'accorde avec la réalité et s'il ne convient pas de pondérer la thèse d'un goût de Descartes pour l'aventure, y compris pour l'aventure intellectuelle.

1 R. Descartes, Discours de la méthode, AT, VI, p. 10. Nous nous référons ici, comme ensuite, à l'éd. suivante, désignée par le sigle AT : CEuvres de Descartes, éd. Ch. Adam et P. Tannery, Paris, Léopold Cerf, 1897-1913, 13 vol. ; rééd. Paris, Vrin/CNRS, 1964-1974, 11 t. en 13 vol.

Ibid., p. 67.

AT, I, p. 570-571.

Ch. Péguy, Note conjointe sur M. Descartes et la philosophie cartésienne, CEuvres en prose, t. III, Paris, Gallimard, « Bibliothèque de la Pléiade », 1992, p. 1280.

5 G. W. Fr. Hegel, La Philosophie moderne, Leçons sur la philosophie de l'histoire. Tome VI, trad. et reconstitution du cours de 1825-1826 par P. Garniron, Paris, Vrin, 1985, p. 1384. 
Si les termes de prudence et d'assurance reviennent très fréquemment sous sa plume, celui d'aventure n'apparaît, à notre connaissance, dans le corpus cartésien que deux fois, aux articles 94 et 147 des Passions de l'âme. Descartes y évoque ces passions de toutes sortes que causent en nous des « aventures étranges " que nous éprouvons comme à distance et par procuration dans les représentations qu'en offrent les pièces de théâtre ou bien les romans : ne pouvant directement nous nuire, elles "semblent chatouiller notre âme en la touchant". Rare sous sa plume, le terme voit ainsi son emploi restreint au domaine des fictions littéraires qui semblent s'interposer entre nous et l'aventure comme pour mieux la tenir à distance ou pour lui dénier toute réalité vécue. Et le Discours de la méthode d'ajouter déjà en forme de discrédit que les récits d'événements imaginés ou réels, a fortiori d'aventures, sont non seulement trompeurs mais dangereux car

ceux qui règlent leurs mœurs par les exemples qu'ils en tirent sont sujets à tomber dans les extravagances des paladins de nos romans, et à concevoir des desseins qui passent leurs forces.

Si nous définissons l'aventure comme une suite accidentelle d'événements extraordinaires aux rebondissements heureux ou funestes dans laquelle on se trouve impliqué volontairement ou non, elle renvoie, selon les termes de Furetière, à "ce qui est au pouvoir du hasard, de la fortune" ${ }^{7}$. Il apparaît alors que l'idée d'aventure se fonde sur celle de fortune laquelle désigne la cause imaginaire de ce qui arrive par hasard ou plus simplement même « ce qui arrive par hasard, qui est fortuit et imprévu" ». Or Descartes traite souvent de la fortune. Aussi nous semble-t-il loisible de cerner une conception cartésienne de l'aventure à partir de nombreuses considérations de Descartes sur la fortune, dont il dit notamment que les âmes vulgaires attendent vainement d'elle une souveraine félicité que nous ne saurions qu'acquérir nous-mêmes et ne tenir que de nous', ajoutant: «J'ai tâché de conduire ma vie en telle sorte, qu'elle [la fortune] n'eût sur moi aucun pouvoir ${ }^{10}$. " Nous verrons alors que ces considérations autorisent à conclure à une certaine disqualification cartésienne de l'idée d'aventure qui pondèrera l'image d'un Descartes au tempérament et à l'esprit aventureux.

\section{Du grand livre du monde au « désert » hollandais}

Nous considérerons que l'aventurier, en quête de biens de la fortune tels que gloire, honneurs ou richesses, vit dans l'attente de l'imprévu alors que l'aventureux accepte de s'exposer à la fortune en prenant des risques. Le premier cède aux charmes de la fortune, le second consent à s'y soumettre. Or il nous semble

R. Descartes, Discours de la méthode, AT, VI, p. 6-7.

A. Furetière, Dictionnaire universel, La Haye, A. et R. Leers, 1690, s.v. « Adventure ". Ibid.

R. Descartes, Lettre à Élisabeth, 21 juillet 1645, AT, IV, p. 252.

10 Id., Lettre à Chanut, 31 mars 1649, AT, V, p. 328. 
que Descartes n'a jamais voulu vivre ni en aventurier ni aventureusement. Objectera-t-on que Descartes s'est engagé comme soldat et que ce choix est manifestement celui d'une existence sans assurance? Encore faut-il examiner ce qui l’y pousse, car Descartes n’intègre des armées que pour voyager, être spectateur du monde, et étudier le genre humain. Ainsi son premier biographe, Adrien Baillet, affirme qu' "il considérait ses campagnes comme de simples voyages ": "il ne se servait de la bandoulière que comme d'un passeport qui lui donnait accès jusqu'au fond des tentes et des tranchées, pour mieux satisfaire sa curiosité $^{11}$ ». L'expérience du monde n'est pas pour Descartes une quête héroïque et il ne s'agit nullement de chercher à se mettre en danger. Elle n'est pas plus objet de divertissement et désir de rompre avec un quelconque ennui. Elle est d'abord objet d'étude ; l'expérience du monde sera motif à réflexion.

Si Descartes se jette dans le grand livre du monde, ce n'est donc point pour y courir l'aventure, en attendre une quelconque gloire ou encore d'extraordinaires rencontres, mais pour continuer par de nouvelles voies la seule chose qui compte à ses yeux, s'instruire. Il s'agit de se déprendre de l'amas confus de connaissances qui ont cours dans les écoles et dans les livres tout autant que de ces opinions héritées d'us et coutumes que nous sommes, depuis notre plus jeune âge, habitués à suivre sans distance critique. Descartes entend se détacher de la culture savante et livresque tout autant que d'une culture, prise dans un sens plus large, que nous n'avons pas à proprement parler apprise mais qui n'en forge pas moins nos manières de penser, de sentir et d'agir. À cet effet, le grand livre du monde s'ouvrira utilement sur les vérités que nous sommes en mesure de recueillir de l'observation des autres et de l'étude de leurs mours.

Descartes espère alors apprendre plus de la vie qu'il n'a pu le faire des livres et de ses maîtres. Le voyage sera ainsi une alternative aux études et leur prolongement. Il conviendra " partout à faire telle réflexion sur les choses qui se présentaient que j’en pusse tirer quelque profit ${ }^{12}$ ". Le profit escompté se mesurera en termes de connaissance et sera motivé par « un extrême désir d'apprendre à distinguer le vrai d'avec le faux, pour voir clair en mes actions, et marcher avec assurance en cette vie ${ }^{13} "$. Le choix de la métaphore du livre pour désigner l'expérience du monde prend ici tous son sens. Il s'agit de s'en instruire, d'y faire provision de connaissances. D'une part, le mouvement ad extra dans le grand livre du monde n'a jamais pour but qu'un mouvement ad intra de retour réflexif en soi-même et la recherche solitaire de la vérité. D'autre part, cette réflexion est elle-même subordonnée au désir d'une marche assurée dans la con-duite de sa vie aux antipodes de l'inconstance et de l'incertitude d'une vie aventureuse.

La recherche de la vérité, la décision d'étudier désormais en soi-même, dont l'expérience du monde est l'antichambre, va alors exiger un certain confort de vie. Descartes cherche à vivre commodément. Cette quête de commodité motive

11 A. Baillet, La Vie de Monsieur Descartes, II, 4, Paris, D. Horthemels, 1691, t. I, p. 98-99.

12 R. Descartes, Discours de la méthode, AT, IV, p. 9.

13 Ibid., p. 10. 
jusqu'aux résolutions de la morale par provision exposée dans la troisième partie du Discours de la méthode:

Et enfin, comme ce n'est pas assez, avant de commencer à rebâtir le logis où on demeure, que de l'abattre, et de faire provision de matériaux et d'architectes, ou s'exercer soi-même à l'architecture, et outre cela d'en avoir soigneusement tracé de dessin, mais qu'il faut aussi s'être pourvu de quelque autre où on puisse être logé commodément pendant le temps qu'on y travaillera."

Vivre le plus commodément possible pour cultiver sa raison conduit Descartes à faire le choix d'une vie retirée et paisible en Hollande. Cet unique objectif précipitera son départ lors d'un de ses rares séjours en France, en raison de sa crainte des désordres de la Fronde. Mêlé de prudence, ce souci le fera encore longuement hésiter à rejoindre la cour de Christine de Suède. Dans une lettre de mai 1631, Descartes décrit les charmes d'une solitude inviolée, alliés à une infinité de commodités indispensables à la vie, dont il jouit dans le plus parfait anonymat, au milieu de l'affairement et de la confusion du peuple d'Amsterdam, et qu'au-cun couvent de capucins ou de chartreux, qu'aucune des plus belles demeures de France et d'Italie réunies, ni aucune maison des champs ne sauraient si bien lui garantiri $^{15}$. Dans cette lettre, qui transpose la description d'Amsterdam en images bucoliques, Descartes insiste sur l'entière liberté, le repos, la sécurité et la parfaite quiétude qu'il y a trouvés et que rien au monde ne pourrait le décider à quitter. Il fera de la Hollande et de ses villes ce qu'il appelle lui-même son désert ${ }^{16}$, au sens d'un lieu propice à la méditation et la retraite spirituelle.

C'est ce même terme que Descartes choisira à la toute fin de la troisième partie du Discours de la méthode pour vanter, à nouveau, les avantages de sa vie hollandaise : « sans manquer d'aucune des commodités qui sont dans les villes les plus fréquentées, j’ai pu vivre aussi solitaire et retiré que dans les déserts les plus écarté $^{17}$ ". Descartes ne sera dans son élément que dans son désert. La dernière lettre qu'il écrivit nous le rappelle avec des accents rétrospectivement testamentaires. Depuis Stockholm, où il ne va pas tarder à trouver la mort, il déclare à Brégy, ambassadeur de France en Pologne, le 15 janvier 1650 :

Mais je vous jure que le désir que j’ai de retourner dans mon désert, s'augmente tous les jours de plus en plus. [...] je ne suis pas ici dans mon élément, et je ne désire que la tranquillité et le repos, qui sont les biens que les plus puissants rois de la terre ne peuvent pas donner à ceux qui ne les savent pas prendre d'eux-mêmes. ${ }^{18}$

14 Ibid., p. 22. Nous soulignons.

15 Id., Lettre à Guez de Balzac, 5 mai 1631, AT, I, p. 202-204.

16 Id., Lettre à Chanut, mai 1648, AT, V, p. 183.

17 Id., Discours de la méthode, AT, VI, p. 31.

18 AT, V, p. 467. 
Ce goût de vivre comme au désert est contemporain de tout un courant traversant le premier $\mathrm{XVII}^{\mathrm{c}}$ siècle, celui d'une spiritualité chrétienne en train de se laïciser, sortant de l'enceinte des couvents et des monastères. L'espace claustral se fait intérieur et sa clôture immatérielle. Il devient celui de l'âme. Cet espace intérieur s'appuie sur un temps particulier, celui des retraites spirituelles. Le paradigme de la clôture recoupe celui du désert où l'âme du chrétien s'isole pour se mettre elle-même à l'épreuve, imitant et revivant la retraite du Christ demeuré quarante jours au désert. Les manuels de dévotion connaissent une vogue considérable. Ils sont destinés à un large public soucieux de sa spiritualité, préoccupé de conquérir son intériorité. Descartes partage avec l'esprit de dévotion et la quête de sainteté qui animent ce siècle très chrétien, où l'homme intérieur, cher à Saint Paul, est à l'honneur, une certaine forme, sinon d'aversion, du moins de distance salutaire envers le monde marqué du sceau de la vanité et de la confusion. L'incessante agitation du monde requiert alors, comme un mal appelle son antidote, de se convertir à soi et d'entrer en soi-même. Le mot d'ordre lâché par saint Augustin dans son De vera religione: « Noli foras ire, in te ipsum redi, in interiore homine habitat veritas " ", tiré d'une traduction latine de la lettre de Paul aux Éphésiens, pourrait servir à caractériser un des traits dominants du courant de dévotion qui se fait jour du temps de Descartes. Le Discours de la méthode ne symbolise-t-il admirablement pas, avec l'image fameuse du poêle, cette clôture intérieure qui est en réalité celle de l'âme recluse en ellemême, se tenant à l'abri de l'écume bruyante des jours? Descartes écrit alors : «Je demeurais tout le jour enfermé seul dans un poêle, où j’avais tout le loisir de m'entretenir de mes pensées ${ }^{20}$."

\section{Une discipline de l'action : agir avec prudence}

Dans l'attente de «distinguer le vrai d'avec le faux pour marcher avec assurance en cette vie ", et surtout à défaut d'avoir le loisir et le temps de le faire en chaque circonstance, il faudra se pourvoir de règles pour faire face aux urgences de la vie. Il convient de se donner des maximes qui, faute de pouvoir réduire la part d'aléas de l'existence, nous permettront de nous mettre à l'abri d'encourir de trop grands risques. La morale par provision de la troisième partie du Discours de la méthode est bien à cet égard un éloge, non de l'aventure, mais de la prudence et de la modération. En matière d'action il faut faire preuve, tout autant chez Descartes que chez Aristote, de phronêsis, de sens de la médiété, de juste mesure dans l'action droite et dans la conduite de nos affaires :

La première [maxime] était d'obéir aux lois et aux coutumes de mon pays, retenant constamment la religion en laquelle Dieu m'a fait la grâce d'être instruit dès mon

\footnotetext{
Augustin, De vera religione, 39, 72 : « Ne t'égare pas au dehors, rentre en toi-même, c'est dans l'homme intérieur que loge la vérité ».
}

20 Discours de la méthode, AT, VI, p. 11. 
enfance, et me gouvernant en toute autre chose suivant les opinions les plus modérées et les plus éloignées de l'excès qui fussent communément reçues en pratique par les mieux sensés de ceux avec lesquels j’aurais à vivre. ${ }^{2}$

Il s'agit, en suivant les opinions les plus modérées, de se garder de toute forme d'excès et en particulier d'erreurs excessivement préjudiciables, si les opinions adoptées venaient à se révéler fausses. Si nous venions à nous être trompés ce ne serait alors que modérément; nous serions moins éloignés de la vérité qu'en ayant opté pour un extrême. Descartes expose ici une théorie du milieu, du choix de la position médiane entre deux extrêmes comme étant la moins dangereuse. Et pour agir avec la plus grande prudence, il conviendra aussi de ne faire aucune promesse, vou ou contrat, car rien au monde ne demeure jamais en même état ${ }^{22}$. Dans la seconde maxime, souvent dite de la constance, là où un esprit aventureux accepterait sans réserve le caractère incertain des événements de la vie, Descartes se propose, pour en conjurer le caractère imprévisible, de traiter ce qui se présente comme douteux et simplement probable à la manière de ce qui est certain. Il s'agit de ne laisser sur soi aucune prise à ce qui advient, la vie n'est pas une aventure :

Ma seconde maxime était d'être le plus ferme et le plus résolu en mes actions que je pourrais, et de ne suivre pas moins constamment les opinions les plus douteuses lorsque je m’y serais une fois déterminé, que si elles eussent été très assurées. ${ }^{23}$

Enfin, se vouer à l'aventure c'est toujours désirer en quelque manière des choses qui ne dépendent pas de nous. C'est fatalement se soumettre à l'empire de la fortune. Or la morale cartésienne - aux accents sur ce point stoïciens - nous invite précisément à nous y soustraire : "Ma troisième maxime était de tâcher toujours plutôt à me vaincre que la fortune, et à changer mes désirs que l'ordre du monde ${ }^{24}$." L'observation de cette maxime a pour but d'harmoniser les relations de la volonté et du désir. Cela exige que nous apprenions à désirer, en ne désirant plus tout et n'importe quoi mais en réglant nos désirs sur notre raison. Rien n'est pire que de laisser nos désirs s'imposer, nous en imposer. Il appartient à la volonté d'apprivoiser nos désirs jusqu'à ne désirer que ce que nous voulons et à ne vouloir que ce que la raison nous présente comme dépendant entièrement de nous de sorte que nous soyons assurés de pouvoir le réaliser.

Dépendent entièrement de nous, à condition bien évidemment d'en faire convenablement usage, notre volonté, nos pensées, nos désirs, nos passions. « Il n’y a rien qui soit entièrement en notre pouvoir que nos pensées ", insiste

21 Ibid., p. 22-23.

22 Ibid., p. 24.

23 Ibid.

24 Ibid., p. 25 
Descartes $^{25}$. Ne dépend pas de nous l'étendue incommensurable des choses qui ne dépendent que de la nature dont nous ne sommes qu'un minuscule rouage. Reste un sous-ensemble d'actions extérieures qui, dépendant de causes physiques, dépendent aussi de notre volonté. Mais pour les choses qui ne dépendent aucunement de nous, "tant bonnes qu'elles puissent être, on ne doit jamais les désirer avec passion ${ }^{26}$ ", et pour celles qui ne dépendent pas seulement de nous ni d'autrui, nous devrons « exactement distinguer en elles ce qui ne dépend que de nous, afin de n'étendre notre désir qu’à cela seul ${ }^{27}$ ». S'interdire la passion de désirer des choses indépendantes de notre volonté, qui adviennent selon l'ordre extérieur des événements, est-ce autre chose que renoncer à la passion de l'aventure parce qu'elle nous expose au risque de voir nos espérances déçues par l'avènement de choses que nous ne désirons pas? À l'emprise de la fortune, Descartes entend opposer une emprise sur nos passions, car désirer avec passion des choses pouvant ne pas arriver nous expose à une affliction d'autant plus grande que notre désir en aura été ardent, alors que " pourvu que notre âme ait toujours de quoi se contenter à son intérieur, tous les troubles qui viennent d'ailleurs n'ont aucun pouvoir de lui nuire ${ }^{28} "$.

La condamnation de la fortune, déjà à l'œuvre dans le Discours de la méthode, trouve ampleur et puissance théorique à l'article 145 des Passions de l'âme. Descartes y considère que la croyance en la fortune nous fait croire possibles des choses qui ne le sont point et qui nous animent de vains désirs, puisque nous ne pouvons désirer que ce que nous estimons en quelque façon être possible. Or la croyance en la fortune fausse notre jugement sur ce qui est possible et par là-même sur ce qui est désirable. Comme pour être heureux il convient de ne désirer que ce que notre raison nous présente comme possible, il faut procéder à une réduction du champ de ce qui est désirable en le purgeant des chimères de l'imagination qu'entretiennent l'idée de fortune et une quête vaine d'aventures qui ne viendront pas. En rédigeant les articles 145 et 146 des Passions de l'âme, Descartes finit par arracher à la fortune son masque "pour la détruire comme une chimère qui ne vient que de l'erreur de notre entendement ${ }^{29} »$. Il faut d'autant moins attendre de la fortune qu'elle n'est rien. Descartes nous donne ainsi motif à ne pas ménager nos efforts pour faire le meilleur usage de notre liberté, sans rien attendre des choses qui ne dépendent pas de nous :

Il faut donc entièrement rejeter l'opinion vulgaire qu'il y a hors de nous une fortune qui fait que les choses arrivent ou n'arrivent pas, selon son plaisir, et savoir que tout est conduit par la Providence divine, dont le décret éternel est tellement infaillible et

25 Ibid. ; Lettre à Reneri pour Pollot, avril ou mai 1938, AT, II, p. 36.

26 Les Passions de l'âme, AT, XI, art. 145, p. 437.

27 Ibid., art. 146, p. 439.

28 Ibid., art. 148, p. 442

29 Ibid., art. 145, p. 438. 
immuable qu'excepté les choses que ce même décret a voulu dépendre de notre libre arbitre, nous devons penser qu'à notre égard il n'arrive rien qui ne soit nécessaire et comme fatal, en sorte que nous ne pouvons sans erreur désirer qu'il arrive d'autre façon. ${ }^{30}$

Le goût de l'aventure s'explique par une croyance dans le caractère fortuit de toutes choses, nous les présentant illusoirement comme possibles alors que la providence divine rend les unes entièrement impossibles et les autres absolument nécessaires. Si nous méditions cette vérité, nous n’attendrions rien d’autre que ce que Dieu veut, bien que nous l'ignorions avant qu'il ne s'accomplisse. Au vain goût de l'aventure, nous substituerions notre soumission à l'ordre divin. Nous ne tiendrions plus abusivement pour imprévu ce qui n'est en réalité qu'imprévisible à nos yeux. Nous cesserions de rêver d'aventures dont l'imagination n'est jamais que l'effet de l'ignorance dans laquelle nous sommes de ce qui est ordonné de toute éternité. L'idée d'aventure suppose en effet qu'il n'y a rien d'imprévisible qui ne soit imprévu, alors que la providence nous fait connaître que rien n'est imprévu dans ce qui nous apparaît comme imprévisible.

\section{Une discipline de la pensée : penser avec méthode}

Pour autant ne doit-on pas admettre que la vie intellectuelle reste pour Descartes le théâtre d'aventures intérieures ? Elle l'est, en réalité, probablement moins que nous sommes souvent tentés de le supposer en songeant au grand renversement qu'opère au moyen du doute radical l'acte inaugural de la philosophie cartésienne. Car le doute cartésien est plus un dispositif savamment orchestré qu'une entreprise aventureuse dont Descartes ignorerait l'issue avant d'y entrer ${ }^{31}$. L'image que Descartes donne de lui-même d'« un homme seul qui marche dans les ténèbres " ", qui perd pied comme s’il était " tombé dans une eau très profonde » au risque de se noyer ${ }^{33}$, qui largue les amarres et se dépouille de toutes ses anciennes opinions les mieux ancrées en son esprit sans garantie de rejoindre la terre ferme de la certitude, qui se trouve en proie à l'adversité d'un Dieu supposé trompeur puis d'un malin génie, relève d'une certaine dramatisation littéraire qu'il ne convient pas de prendre au pied de la lettre. Si l'espoir de la vérité semble un moment s'avancer sur une corde surplombant un

30 Ibid., art. 146, p. 439.

31 Sur la dramatisation de l'entreprise intellectuelle et « une ligne d'interprétation qu'on pourrait dire déflationniste" des risques encourus dans la recherche cartésienne de la vérité, voir D. Kambouchner, "Descartes : la certitude au risque de la psychose? ", Séminaire Champ lacanien, $\mathrm{n}^{\circ} 116$, juin 2017 , p. 7-25.

32 Discours de la méthode, AT, VI, p. 16.

33 Méditations métaphysiques, II, AT, IX, 1, p. 19. 
abîme, il faut toutefois se déprendre d'une lecture inflationniste des raisons de douter $^{34}$.

En réalité, la vérité n’est jamais ébranlée dans ses fondements, de sorte que la perspective d'un accès assuré à la vérité n'est jamais vraiment menacée. L'incipit des Méditations métaphysiques ne manquera pas de le laisser entrevoir de manière nette. En déclarant en effet : «Il y a déjà quelque temps que je me suis aperçu que, dès mes premières années, j’avais reçu quantité de fausses opinions pour véritables ", Descartes suppose et indique de façon manifeste qu'il dispose de la capacité effective de distinguer le vrai du faux avant même la mise en ouvre de la grande machinerie et de la mise en scène du doute qui ne consistera pas dès lors à remettre en cause la possibilité de connaître la vérité mais seulement à faire comme si, pour un temps, plus aucune vérité ne pouvait être assurée.

Le doute volontaire, méthodique, hyperbolique est avant toute chose une fiction très savante et très construite par laquelle Descartes feint plus l'aventure de la pensée qu'il ne s'y expose réellement. Alors qu’on pourrait se croire submergé par l'ampleur croissante des vagues successives qui alimentent le tourbillon du doute, la terre ferme du cogito n'est jamais bien loin à supposer qu'elle ait même un instant été perdue de vue. Il y a ainsi une distance entre l'expérience de pensée des Méditations et leur auteur, qui fait qu’il ne vit pas l'aventure du doute mais qu'il l'orchestre. Descartes sait parfaitement où il va et où le doute doit le conduire au terme d'une marche bien plus assurée qu'il ne pourrait sembler. Loin d'une aventure, le doute est bien plutôt une grande fiction, un jeu de l'esprit, une orchestration complexe, une mise en scène aux accents baroques, une expérience de pensée vertigineuse et un puissant dispositif pédagogique visant à préparer le lecteur à l'assimilation des vérités métaphysiques.

À ce titre, tout n'est qu'affaire de méthode. La première règle de la méthode nous invite à " éviter soigneusement la précipitation et la prévention ". ". Pour se garder de trébucher, il faut n'avancer que prudemment, pas à pas, quitte à ne progresser que lentement. Ceux qui négligent de suivre une méthode et un chemin qui va des choses les plus faciles aux plus difficiles conduisent en vain leur pensée à l'aventure et perdent leur temps ainsi que leur énergie ${ }^{36}$. La science moderne ne doit pas ressembler à " ces anciennes cités qui, n’ayant été au commencement que des bourgades, sont devenues par succession de temps de grandes villes ». Les éléments qui les composent sont irréguliers, sans ordre, et " on dirait que c'est plutôt la fortune que la volonté de quelques hommes usant de raison, qui les a ainsi disposés ". La vérité ne se connait pas par hasard, tout n'est dans le domaine de sa quête que maîtrise de nos pensées et question

34 Voir F.-X. de Peretti, "Essai de lecture déflationniste du doute ", Certitude, évidence et vérité chez Descartes. La question du fondement cartésien de la connaissance, Lecce, Conte, 2015, p. $97-161$.

35 R. Descartes, Discours de la méthode, II, AT, VI, p. 18. Nous soulignons.

36 Id., Regulae, II, AT, X, p. 362 et 364 ; IV, p. 371 ; V, p. 380 ; IX, p.401 ; XII, p. 427. 
d'ordre. La science cartésienne est une construction méthodique, pas une aventure.

\section{Entre providence et règne de l'ordinaire : le contentement intérieur}

Dans un double sens fécond, la science et l'ordre de la pensée cartésienne instaurent le règne de l'ordinaire, qui signifie à la fois l'ordre et la banalité qui en résulte par l'éviction de tout secret ou mystère, mettant fin au règne poétique de l'admiration de la nature. L'admiration aura basculé du côté de la science. Ce qui devient véritablement admirable est moins ce que nous connaissons que de le connaître. Dans un monde dont les phénomènes sont réglés par les lois immuables de la nature et les événements de l'histoire par les décrets de la providence, il ne reste aucun motif de se mettre en quête de l'extraordinaire.

Aussi le vrai contentement cartésien sera-t-il intérieur. Descartes l'appelle générosité et celle-ci consiste dans l'usage de notre libre-arbitre, dans une volonté ferme et constante d'exécuter tout ce que nous jugeons être le meilleur, et dans l'emploi de la force de notre entendement à en bien juger. Ce qui en définitive prévaut pour nous rendre heureux sur toutes considérations contingentes tient à l'usage de notre bonne volonté. Ce bonheur du généreux que lui accorde, et auquel suffit, l'exercice de sa vertu a l'insigne caractéristique d'être inaltérable même dans le malheur. Il nous accorde une tranquillité d'âme inexpugnable qui ne reçoit des passions et des aléas de l'existence aucune incommodité. Il en ira de la vie comme il en va du théâtre, où nous pouvons éprouver une joie intellectuelle bien que les passions qui s'y jouent inspirent de la tristesse ${ }^{37}$. Empruntant le terme au lexique de la théologie, Descartes n’hésite pas à qualifier ce bonheur tout intérieur qui naît de notre seule vertu de " béatitude ${ }^{38}$ ".

Ultime argument pour se détacher de la fortune : les choses qui sont en son pouvoir ou que notre imagination juge telles sont si peu comparées à la vie éternelle qu'il y a matière à les regarder avec mépris et à se détacher des événements de cette vie ${ }^{39}$. À la date du 18 mai 1645, Descartes déclarait encore à Élisabeth considérer que le théâtre nous donne un exemple de ce que peut être notre détachement à l'égard des événements du monde qui au regard de l'éternité font figure de comédie ${ }^{40}$. La frontière entre ce qui arrive dans le monde et ce qui n'arrive qu'en fiction semble s'estomper, et les incertitudes qui pourraient faire de notre expérience immanente du monde une aventure cèdent le pas chez Descartes à la seule quête qui vaille et à laquelle il consacrera sa vie, celle de la certitude que satisfera la connaissances de vérités dont la nature immuable de Dieu nous asure la permanence.

37 Id., Les Passions de l'âme, AT, XI, , art. 147, p. 440-441 ; Lettre à Élisabeth, 18 mai 1645, AT, IV, p. 202-203.

38 Id., Lettre à Élisabeth, 18 août 1645, AT, IV, p. 277.

39 Id., Lettre à Élisabeth, 15 septembre 1645, AT, IV, p. 292.

40 Id., Lettre à Élisabeth, 18 mai 1645, AT, IV, p. 202. 
François-Xavier de Peretti Aix-Marseille Université Institut d'Histoire de la Philosophie (E.A 3276) 